LA W RENCE LIVERMORE N A TIO NAL LABORATORY

DOE Computer Graphics Forum 2013 Site Survey

E. S. Brugger, R. R. Springmeyer, D. E. Laney

April 15, 2013 
This document was prepared as an account of work sponsored by an agency of the United States government. Neither the United States government nor Lawrence Livermore National Security, LLC, nor any of their employees makes any warranty, expressed or implied, or assumes any legal liability or responsibility for the accuracy, completeness, or usefulness of any information, apparatus, product, or process disclosed, or represents that its use would not infringe privately owned rights. Reference herein to any specific commercial product, process, or service by trade name, trademark, manufacturer, or otherwise does not necessarily constitute or imply its endorsement, recommendation, or favoring by the United States government or Lawrence Livermore National Security, LLC. The views and opinions of authors expressed herein do not necessarily state or reflect those of the United States government or Lawrence Livermore National Security, LLC, and shall not be used for advertising or product endorsement purposes.

This work performed under the auspices of the U.S. Department of Energy by Lawrence Livermore National Laboratory under Contract DE-AC52-07NA27344. 


\section{DOE Computer Graphics Forum 2013 Site Survey}

Site Name: Lawrence Livermore National Laboratory

Site Division or Group: Computation: Data Group, Information Management and Graphics Group, Center for Applied Scientific Computing (CASC)

Site Representative: Eric Brugger, Richard Cook, Becky Springmeyer

\section{Site Representative Contact Information:}

Eric Brugger, P.O. Box 808, L-170, Livermore, CA 94550, (925) 423-1293, brugger1@1lnl.gov

Becky Springmeyer, P.O. Box 808, L-557, Livermore, CA 94550, (925) 423-0794, springme@1lnl.gov

Rich Cook, P.O. Box 808, L-557, Livermore, CA 94550, (925) 423-9605, rcook@1lnl.gov

LLNL-TR-635217

This work was performed under the auspices of the U.S. Department of Energy by Lawrence Livermore National Laboratory under contract DE-AC52-07NA27344. Lawrence Livermore National Security, LLC

\section{Group Overview}

\section{Mission:}

The Data group provides data analysis and visualization support to its customers. This consists primarily of performing data analysis and visualization for customers, assisting customers with the use of visualization and analysis tools, and the development of VisIt, a data analysis and visualization tool.

The Information Management and Graphics Group supports and develops tools for accessing, displaying, and understanding large, complex data sets. Activities include large scale data exploration; video production; supporting graphics libraries and tools; maintaining PowerWalls and other displays; developing software for searching and managing scientific data, and providing easy-to-use interfaces to HPC resources.

Researchers in the Center for Applied Scientific Computing (CASC) work on various projects including the development of visualization techniques for large scale data exploration that are funded by the ASC program, among others. The researchers also have LDRD projects and collaborations with other lab researchers, academia, and industry.

\section{Past Year's Activities:}


The IMG group is located in the simulation facility that houses Sequoia, Vulcan, Zin (TLCC2), and many other platforms. The facility includes visualization theaters, a visualization computer floor and deployment workshop, and video production labs. We provided the traditional graphics group consulting and video production support. We maintained five PowerWalls and many other displays. We supported edge, a 216node Westmere/IB cluster with NVIDIA Tesla M2050 GPUs and more than 20TB of memory, providing a visualization production server on our unclassified network. We continue to support a 576-node Opteron/IB cluster with $72 \mathrm{~TB}$ of memory on our restricted network, as well as several smaller machines to drive the PowerWalls. The visualization production systems include NFS servers to provide dedicated storage for data analysis and visualization.

The ASC projects have delivered new versions of visualization and scientific data management tools to end users and continue to refine them. VisIt had multiple releases during the past year. We released new versions of Hopper, a Java application for managing and transferring files. We released versions of Lorenz, also known as MyLC, a web-based interface to our HPC resources and services. We continue to use and develop Blockbuster and Telepath. Both the VisIt and IMG teams were engaged in a variety of movie production efforts during the past year in addition to the development tasks. The research team is exploring applications of high dimensional functional analysis for NIF applications, data compression for mitigating data movement bottlenecks in HPC codes, and worked with domain scientists to apply both off-the-shelf and custom methods for data analysis.

Information on these tools and efforts can be found on our web pages http://www.llnl.gov/icc/sdd/img/infrastructures.shtml and at the VisIt site: https://visit.llnl.gov.

\section{Plans and Priorities:}

We will release new versions of the various in-house tools we support. We will procure and deploy new visualization platforms with GPUs. We will continue to provide consulting and support services in graphics and video production.

Research into visualization techniques continues, funded largely by sources other than the ASC program. The research priorities for next year include topological analysis for scientific computing, particle visualization, data compression, uncertainty quantification, and mentoring students in various areas related to the above.

\section{Funding Sources}

The IMG Group is funded by ASC as well as other institutional sources. CASC is funded by LDRD, ASC, other LLNL projects, and outside sponsors. The Data group is funded by ASC, SciDAC, and NEAMS.

\section{Current Resources}

\section{Hardware:}

Compute servers: See http://www.llnl.gov/computing/hpc/resources/ for details on the compute servers and storage systems. 
Production visualization servers: We have one unclassified and two classified production visualization servers, one small development server with GPUs (M2070s), three classified PowerWalls with dedicated machines to drive them, and two unclassified PowerWalls with dedicated machines to drive them.

Graph Linux production cluster: 576 nodes $(242.0 \mathrm{GHz}$ Opteron (quad-socket, six core Istanbul),128GB RAM per node), InfiniBand, shared Lustre disk, total of $72 \mathrm{~TB}$ memory

Edge Linux production cluster: 216 nodes (12 2.8 GHz Westmere (dual-socket, six core), 96GB RAM per node), InfiniBand, nVidia Tesla M2050, shared Lustre disk, total of 20TB memory

Video production: Our two labs include PCs, Macs, desktop video editors, and assorted recorders, monitors, mixers, and software packages. Visualization developer's lab: Assorted small systems and a 15-panel display driven by multiple Linux clusters.

Desktop systems for visualization consist of diskless and diskfull Linux, Mac and Windows systems, with Linux the being the most common.

\section{Software:}

VisIt, EnSight, IDL, Tecplot, AVS, assorted other utilities and translators, and Blockbuster. SDM software includes Hopper, Chopper, and tools used in the Green Data Oasis.

Blockbuster: http://blockbuster.sourceforge.net/

Hopper: https://computing.1lnl.gov/resources/hopper/

VisIt: https://visit.llnl.gov

\section{Staff:}

In the Information Management and Graphics Group (led by Jeff Long, with Becky serving as vis project and CSSE lead and LC deputy) there are 8 people: four developers working on scientific data management tools and four people on the vis team. In the DATA group (led by Eric) there are 5 people. In CASC there are several visualization and data analysis researchers (Dan Laney, Group Leader), along with ongoing collaborations with several graduate students. No hires are planned for the development groups but we may hire a researcher this year.

\section{Planned Growth}

\section{Hardware Acquisitions}

We plan to acquire a TLCC2 Scalable Unit with some GPUs on the classified network this year. We are also buying a technology refresh cluster with Keplers.

\section{Software Acquisitions}

None are currently on the list, other than GPU-enabled versions of existing packages.

\section{Personnel Acquisitions}


We may hire an additional visualization researcher. No new developer hires are planned.

\section{Additional Comments}

